\title{
Effectiveness of Two Types of Exercises before Classes on Inhibitory Function
}

\author{
Watanabe Noriaki ${ }^{1}$, Tabuchi Hisaaki ${ }^{2}$, Suzuki Satoko ${ }^{3}$, Watanabe Toshiaki ${ }^{2}$, Terasawa Saiki ${ }^{4}$, Nakade Keisuke ${ }^{5}$, Terasawa Koji ${ }^{*}$ \\ ${ }^{1}$ Fujieda Municipal Aoshima primary school; 10 Shimoaoshima Fujieda Shizuoka 426-0035 Japan \\ ${ }^{2}$ Shinshu University, Faculty of Education; 6-Ro Nishinagano Nagano 380-8544 Japan \\ ${ }^{3}$ The University of Melbourne, Graduate School of Education; 32 Lincoln Square North Carlton 3053 VIC Australia \\ ${ }^{4}$ Shinshu University, Faculty of Engineering; 4-17-1 Wakasato Nagano 380-8553 Japan \\ 5Shinshu University, Graduate School of Medcine; 3-1-1 Asahi, Matsumoto, Nagano 390-8621 Japan
}

*Corresponding author: Professor Koji Terasawa, Department of Medicine, Shinshu University, Graduate School of Medcine; 3-1-1 Asahi, Matsumoto, Nagano 390-8621, Japan, Tel: +81-26-238-4213; E-mail: kterasa@shinshu.ac.jp

Received date: February 16, 2016, Accepted date: March 07, 2016, Published date: March 14, 2016

Copyright: (C) 2016 Noriaki W, et al. This is an open-access article distributed under the terms of the Creative Commons Attribution License, which permits unrestricted use, distribution, and reproduction in any medium, provided the original author and source are credited.

\begin{abstract}
The purpose of this study is to find an effective solution to the status quo, children cannot concentrate on the class and behave irrationally; they are emotionally unstable and sometimes interrupt the class and even go away from the class. This is the case recently seen in the many primary schools in Japan. To solve this issue, the author, a teacher in a public primary school, found out an effective impact caused by the physical activities, called GO/NO-GO task, in order to investigate prefrontal cortex development in Japanese children through two types of exercises before classes. As a result, a faster response time and higher accuracy rate for GO/NO-GO tasks were observed. Based on this, since the accuracy rate after the two types of exercises in this study were significantly higher than those after the control activity and reading, it is suggested that two types of exercises were effective in improving inhibitory function.
\end{abstract}

Keywords: GO/NO-GO task; Children's behavior; Exercises; Prefrontal cortex

\section{Introduction}

The increasing issue of problem behaviors in the classroom has been a topic of discussion in some countries. It has been reported that there are some problems such as truancy, bullying, collapse of classroom structure, and so on, among school children in Japan [1]. It has also been reported that recently, the number of children with Attention Deficit/Hyperactivity Disorder (ADHD) and Learning Disorders (LD) has been increasing [2]. However, the cause of these problems has not yet been found.

According to studies in sports science and the relevant pedagogy, doing exercise is often considered an effective method of relieving such behavioral problems in both adolescents and adults. Verret et al. [3] reported that a 10 -week physical activity training program for children with ADHD improved their muscular capacities, motor skills, levels of information processing, and behavior in reports written by parents and teachers. Tantillo et al. [4] presented that the behavioral ADHD traits improved after exercise in children with ADHD. Niederer, Kriemler et al. [5] also suggested that preschool children with greater fitness levels and motor skills have increased spatial working memory and attention (mean age: $5.2( \pm 0.6)$ years, girls: $49.4 \%)$.

Furthermore, Hirano et al. [6] reported that the prefrontal cortex function of children performing a GO/NO-GO task, which was used to evaluate the function of the prefrontal cortex, was significantly improved after a three-day camping excursion that included exercise.
As discussed above, it is apparent that exercise contributes to improved prefrontal cortex function in children. In contrast, it has also been found that some medications that have been advanced for ADHD help improve ADHD symptoms. In addition to the practice of preventive exercise, the intake of supplements with minimal side effects was found effective in preventing problem behaviors. Hirayama et al. [7] demonstrated that the prefrontal cortex function and mental index of children with ADHD who took $200 \mathrm{mg}$ of phosphatidylserine a day for two months significantly improved.

However, rather than taking supplements and/or medications, it is assumed that alternative prevention, such as habitual physical exercise, could have sustainable positive effects on problem behaviors. We, as teachers in Japanese schools, face the harsh reality of children losing their concentration and not being able to sit calmly throughout an entire class. However, the author has realized that exercising before classes helps children concentrate on their studies in class for the whole day.

The purpose of this study was to verify the effect of two types of exercise in which children interact and have physical contact with each other before classes by using the GO/NO-GO tasks.

\section{Method}

The subjects of the study were 34 healthy elementary school students (16 males and 18 females) in the fifth grade, with a mean age ranging between 10 and 11 years $(S D=0.29)$ in 2009 , in Japan. The children and their parents gave written informed consent. In Japanese elementary schools, there is a morning meeting to take attendance, check the children's health, and make announcements. It takes about 25 minutes. During the time of the morning meeting, all 34 subjects 
did one of four activities on one day every other week. The four activities were the morning meeting for the day of the first week (control), reading for the second week, exercise 1 for the third week and exercise 2 for the fourth week. Each activity took 25 minutes, and was conducted from 8:05 to 8:30, before the first class in the morning.

On the reading day, subjects read their favourite books in the classroom, which has recently become a widespread practice in Japanese elementary, junior high and high schools.

On the day for exercise 1, the 34 subjects were divided into two groups. One group stood on one side of an S-shaped territory drawn on the playground. The other group stood on the other side of the same territory. Hopping on one foot, they had to go to the territory of the opposing group and try to capture their treasure. This game is called "S-ken" (Figure 1).

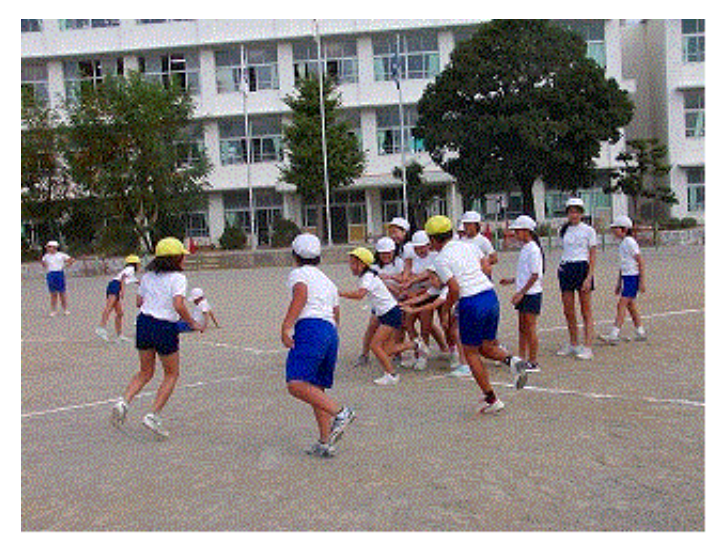

Figure 1: "S-ken" game.

On the day of exercise 2, about 30 subjects lay on their stomachs on mats in the gym, and held hands with one another to form a circle. Then, the remaining four subjects played the role of "demon." The goal of the demons was to break the circle by pulling on the legs of the subjects forming the circle. This game is called "Kabu" (Figure 2).

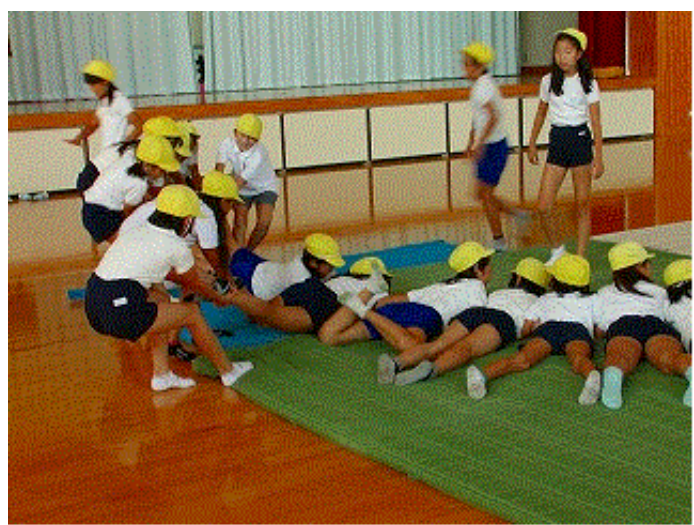

Figure 2: "Kabu" game.

The subjects can enjoy the two exercises, having interaction and physical contact with each other. After each activity, groups of six subjects at a time sat in separate booths in a special classroom and performed the GO/NO-GO task [8,9]. The other 28 subjects waited calmly in their classroom.

\section{Procedure}

Computer-controlled equipment (ME Corporation, Japan) was used to evaluate the GO/NO-GO task in this study. Six booths were placed in a special classroom. Each subject was seated in a booth enclosed on three sides by $45 \mathrm{~cm} \times 45 \mathrm{~cm}$ panels. A stimulus presentation device was set $70 \mathrm{~cm}$ in front of each subject. First, each subject held a rubber ball with their dominant hand. Then, the subject was required to squeeze the ball when the stimulus presentation device produced a light. The stimulus presentation device was adjusted to each subject's eye height in Figure 3.

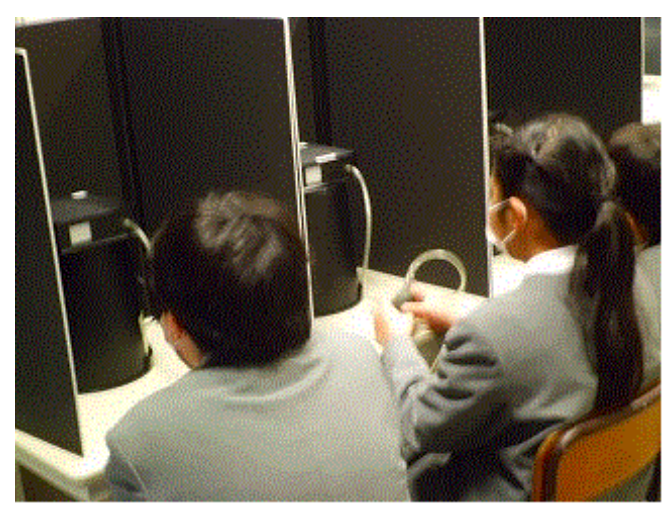

Figure 3: stimulus presentation device.

The stimulus presentation was designed to light up inside a framed rectangle $(2.5 \mathrm{~cm} \times 3.7 \mathrm{~cm})$ at the centre of the device.

\section{GO/NO-GO task procedure}

Masaki's method [10] was used for the GO/NO-GO task. The subjects were instructed to respond to red and yellow light stimuli by squeezing a rubber ball. The GO/NO-GO task in this study consisted of three phases: formation, differentiation and reverse differentiation.

In the formation phase, subjects were instructed to squeeze the rubber ball in response to a red light being turned on. In this phase, it was verified that all subjects were able to follow the instructions and could squeeze the rubber ball. The stimulus was presented five times for 0.5 to 1.5 seconds every three to six seconds.

Next, the subjects moved on to the differentiation phase. This phase entailed a red light or a yellow light being turned on. The instructions were to squeeze the rubber ball in response to only the red light being turned on, but not to squeeze in response to the yellow light. In this phase, red and yellow lights were respectively turned on for 0.5-1.5 seconds ten times each in random order.

In the reverse differentiation phase, the instructions were the opposite of those for the differentiation stage. The subjects were told to squeeze the rubber ball only in response to the yellow light being turned on, but not to squeeze when the red light was turned on.

Illumination of the red and yellow lights was the same as for the differentiation phase. 


\section{GO/NO-GO task analysis}

Response time, which was the time taken to squeeze the rubber ball, was recorded for each phase: formation, differentiation, and reverse differentiation. A total of 40 repetitions were used for analysis, including 20 repetitions each for the differentiation and reverse differentiation phases. All the data was collected to check the accuracy rate for each of the four activities: the morning meeting, which was defined as control, reading, exercise 1 and exercise 2. Response times and accuracy rates were compared between the four activities using one-way ANOVA. Tukey's HSD (honestly significant difference) test was used as post-hoc. A graph was used to compare the accuracy rate clearly between the four activities. Statistical analyses were performed using SPSS statistical packages. The significance level was set at 0.05 .

\section{Results}

During the GO/NO-GO tasks in this study, subjects failed to squeeze the rubber ball when they should have squeezed it or mistakenly squeezed the ball when they should not have. The response time, the number of fails and mistakes were analyzed in the formation, the differentiation and reverse differentiation phases.

Figure 4 shows the response times for the GO/NO-GO task after each activity.

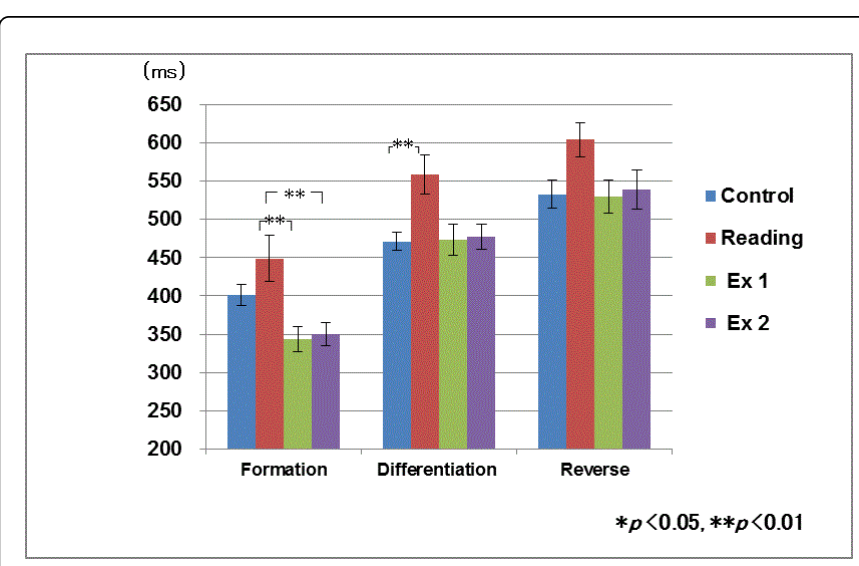

Figure 4: The response times for the GO/NO-GO task after each activity.

There were significant differences between the four activities for each of the three task phases: formation $\left(\mathrm{F}_{(2.676)}=6.3, \mathrm{P}<0.001\right)$, differentiation $\left(\mathrm{F}_{(2.673)}=5.1, \mathrm{P}<0.01\right)$ and reverse differentiation $\left(\mathrm{F}_{(2.673)}=2.8, \mathrm{P}<0.05\right)$. Subsequently, response times after each activity were compared with each other using Tukey's method.

In the formation phase, the average response time of the subjects after reading was $448.9 \mathrm{~ms}$. This was the slowest time, followed by the control activity, exercise 2, and exercise 1. It was found that the response times after exercises 1 and 2 were significantly faster than those after reading (reading vs. exercise $1, \mathrm{P}<0.01$; reading vs. exercise $2, \mathrm{P}<0.01)$.

In the differentiation phase, the average response time of $558.5 \mathrm{~ms}$ after reading was the slowest, followed by exercise 2 , exercise 1 , and the control activity. It was found that the response times after reading were significantly slower than those after the control activity: control vs. reading, $\mathrm{P}<0.01)$.
In the reverse differentiation phase, the average response time of $604.2 \mathrm{~ms}$ after reading was the slowest, followed by exercise 2, exercise 1 , and the control activity. However, there was no significant difference in the response times after the four activities for this phase.

During the GO/NO-GO tasks in this study, subjects sometimes failed to squeeze the rubber ball when they should have squeezed it or mistakenly squeezed the ball when they should not have. The number of fails and mistakes were analysed using one-way ANOVA for the following nine cases in the differentiation and reverse differentiation phases. These analyses below were all conducted after each of the four activities.

Case 1: Number of fails in the differentiation phase, Case 2: Number of mistakes in the differentiation phase, Case 3: Number of fails and mistakes in the differentiation phase, Case 4: Number of fails in the reverse differentiation phase, Case 5: Number of mistakes in the reverse differentiation phase, Case 6: Number of fails and mistakes in the reverse differentiation phase, Case 7: Number of fails in total for the differentiation and reverse differentiation phases, Case 8: Number of mistakes in total for the differentiation and reverse differentiation phases and Case 9: Number of fails and mistakes in total for the differentiation and reverse differentiation phases.

The results revealed significant differences in cases 3, 8 and 9: case 3 $\left.\left(\mathrm{F}_{(2.673)}\right)=14.0, \mathrm{P}<0.001\right)$, case $\left.8\left(\mathrm{~F}_{(2.673)}\right)=6.8, \mathrm{P}<0.001\right)$, and case 9 $\left(\mathrm{F}_{(2.673)}=6.8, \mathrm{P}<0.001\right)$. Figure 5 shows the results for case 3 .

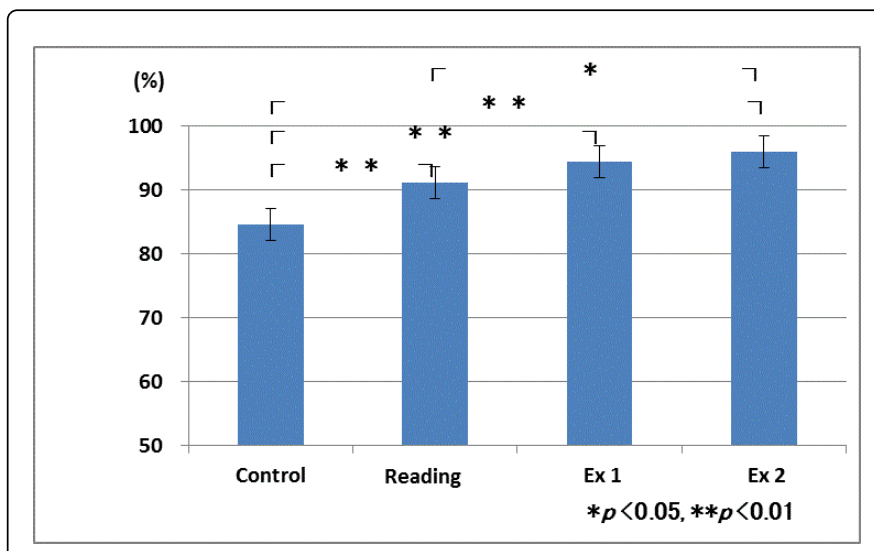

Figure 5: The accuracy rate of case 8 for the GO/NO-GO task after each activity.

The accuracy rate after exercise 2 was $96.0 \%$, followed by exercise 1 , $94.5 \%$, reading, $91.2 \%$, and control, $84.6 \%$. The accuracy rates for exercise 2, exercise 1 and reading were all significantly higher than that for the control activity (control vs. reading, $\mathrm{P}<0.01$; control vs. exercise $1, \mathrm{P}<0.01$; control vs. exercise $2, \mathrm{P}<0.01$ ). Further, the accuracy rate after exercise 2 was significantly higher than that after reading (reading vs. exercise $2, \mathrm{P}<0.05$ ). Figure 6 shows the results for case 8.

The accuracy rate after exercise 2 was $90.2 \%$, followed by exercise 1 , $88.5 \%$, reading, $84.4 \%$, and control, $77.1 \%$. The accuracy rates for exercise 2, exercise 1 and reading were significantly higher than that for the control activity (control vs. reading, $\mathrm{P}<0.01$; control vs. exercise $1, \mathrm{P}<0.01$; control vs. exercise $2, \mathrm{P}<0.01$ ). 


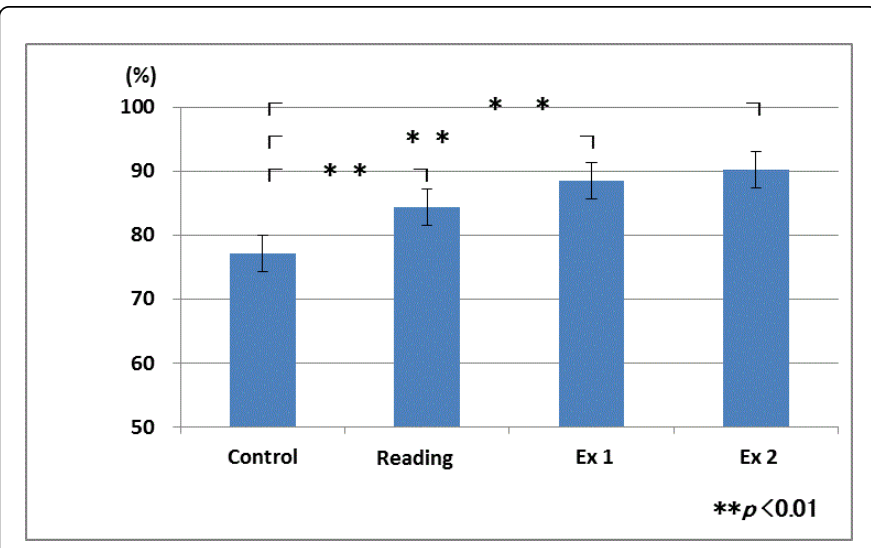

Figure 6: The accuracy rate of case 8 for the GO/NO-GO task after each activity.

The accuracy rate for exercise 2 was $94.5 \%$, followed by exercise 1 , $93.4 \%$, reading, $90.5 \%$, and control, $87.3 \%$. Compared with the control activity, accuracy rates were significantly improved after exercises 1 and 2 (control vs. exercise $1, \mathrm{P}<0.01$; control vs. exercise $2, \mathrm{P}<0.01$ ). Figure 7 shows the results for case 9 .

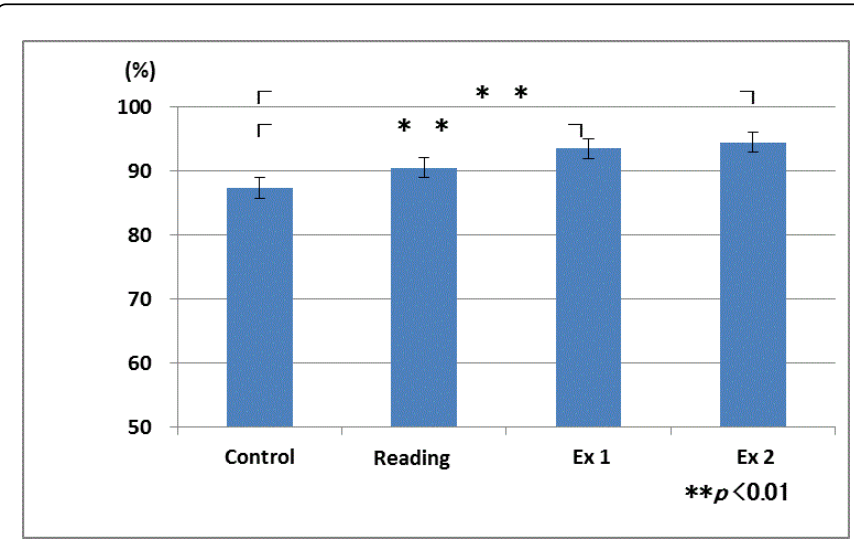

Figure 7: The accuracy rate of case 9 for the GO/NO-GO task after each activity.

\section{Discussion}

The GO/NO-GO task originated in Pavlov's conditioned reflex experiment, which used the sound of bells. Pavlov's concept was developed into the GO/NO-GO task using a light stimulus. Luria et al. [11], who were cerebral physiologists, revealed that the function of the prefrontal cortex is deeply associated with the conditioned reflex. Given its historical background, the GO/NO-GO task is considered a discrimination task, and it is used to evaluate the function of disinhibition of the PFC (prefrontal cortex). Sasaki et al. [12] claimed that "no-go potential" was found in in the dorsolateral parts of frontal lobes in both contra- and ipsilateral hemispheres when human subjects were not supposed to squeeze a rubber ball in a GO/NO-GO task.

Moreover, Sawaguchi et al. [13] revealed that the dorsolateral PFC plays an important role in working memory. The function of working memory is to store a variety of information temporarily and to make final judgments. In addition, Fuster [14] claimed that the inhibitory function is carried out by collaboration between the orbitofrontal cortex, the prefrontal cortex, the basal ganglia, the thalamus and the hypothalamus.

During a GO/NO-GO task, attention control processes are required for both the initiation of action [15-17] and the inhibition of inappropriate responses $[18,19]$. Tamm $[20]$ reported that the GO/NOGO task requires multiple executive functions including working memory, interference avoidance, and the withholding of responses that have been established as proponent response.

In the present study, the increased number of errors suggests that the PFC function involved in working memory may be immature $[21,22]$. Consistent with these previous studies, the present research has used a GO/NO-GO task to verify the effectiveness of two types of exercise over reading or the normal morning meeting (control). Subjects have interaction and physical contact with each other in both exercises.

Compared with the control activity and exercises 1 and 2, the response time after the reading activity was the slowest. Furthermore, although the GO/NO-GO task accuracy rate after reading was higher than that after the control activity, it was lower than those for exercises 1 and 2. In particular, as shown in Figure 2, the accuracy rate after exercise 2 was significantly higher than that after reading in regards to the number of fails and mistakes during the differentiation phase. As shown in Figure 4, the total number of fails and mistakes for the differentiation and reverse differentiation phases reveals that accuracy rates after exercises 1 and 2 were significantly higher than those after the control activity, but there were no significant differences between the control activity and reading. Therefore, post-exercise 1 and 2 results were better than those of post-reading for the GO/NO-GO task.

Nakade et al. [23] and Murata et al. [24] reported in previous GO/NO-GO experiments that response times were reduced and the number of errors decreased when subjects walked an average of 7,000 steps per day for 10 months. It was also reported that when subjects continued this walking exercise for two years, response times became significantly faster and the number of errors significantly decreased.

According to previous studies, a faster response time and higher accuracy rate are considered good results for GO/NO-GO tasks. Based on this, since the accuracy rate after exercises 1 and 2 in this study were significantly higher than those after the control activity and reading, it is believed that exercises 1 and 2 were effective in improving inhibitory function. In the future, the author would like to conduct further research using various methods such as a profile of mood states (POMS), the Stroop Test and the Wisconsin Card Sorting Test to investigate the prefrontal cortex.

\section{References}

1. Haigen Gu, Shu-Ling Lai, Renmin Ye (2011) A cross-cultural study of student problem behaviors in middle schools. School Psychology International 32: 20-34.

2. DuPaul GJ, Gormley MJ, Laracy SD (2013) Comorbidity of LD and ADHD: implications of DSM-5 for assessment and treatment. J Learn Disabil 46: 43-51.

3. Verret C, Guay MC, Berthiaume C, Gardiner P, Béliveau L (2012) A physical activity program improves behavior and cognitive functions in children with ADHD: an exploratory study. J Atten Disord 16: 71-80. 
Citation: $\quad$ Noriaki W, Hisaaki T, Satoko S, Toshiaki W, Saiki T, et al. (2016) Effectiveness of Two Types of Exercises before Classes on Inhibitory

Page 5 of 5

4. Tantillo M, Kesick CM, Hynd GW, Dishman RK (2002) The effects of exercise on children with attention-deficit hyperactivity disorder. Med Sci Sports Exerc 34: 203-212.

5. Niederer I, Kriemler S, Gut J, Hartmann T, Schindler C, et al. (2011) Relationship of aerobic fitness and motor skills with memory and attention in preschoolers (Ballabeina): a cross-sectional and longitudinal study. BMC Pediatr 11: 34.

6. Hirano Y, Shinohara K, Yanagisawa A, Tanaka Y, Nemoto K, et al. (2001) Effects of long-term camp experience has on the brain activity of children. National Olympics Memorial Youth Center Bulletin pp: 261-268.

7. Hirayama S, Terasawa K, Rabeler R, Hirayama T, Inoue T, et al. (2013) The effect of phosphatidylserine administration on memory and symptoms of attention-deficit hyperactivity disorder: a randomised, double-blind, placebo-controlled clinical trial. J Hum Nutr Diet 2: 284-291 .

8. Terasawa K, Tabuchi H, Yanagisawa H, Yanagisawa A, Shinohara K, et al. (2014) Comparative survey of go/no-go results to identify the inhibitory control ability change of Japanese children. BioPsychoSocial Medicine 8: 1-14.

9. Terasawa K, Misaki S, Murata Y, Watanabe T, Terasawa S, et al. (2014) Relevance between Alzheimer's disease patients and normal subjects using go/no-go tasks and Alzheimer assessment scores . Journal of Child and Adolescent Behavior 2:162.

10. Masaki T, Moriyama G (1971) A study on the types of the human higher nervous activity. Tokyo Science University Kiyo 4: 69-81.

11. Luria AR (1966) Higher cortical function in man. London, UK: Travistock.

12. Sasaki K, Gemba H, Nambu A, Matsuzaki R (1993) No-go activity in the frontal association cortex of human subjects. Neurosci Res 18: 249-252.

13. Sawaguchi T, Goldman-Rakic PS (1994) The role of D1-dopamine receptor in working memory: local injections of dopamine antagonists into the prefrontal cortex of rhesus monkeys performing an oculomotor delayed-response task. J Neurophysiol 71: 515-528.
14. Fuster JM (1989) The prefrontal cortex. New York USA: Raven Press.

15. Cabeza R, Anderson ND, Houle S, Mangels JA, Nyberg L (2000) Agerelated differences in neural activity during item and temporal-order memory retrieval: a positron emission tomography study. J Cogn Neurosci 12: 197-206.

16. Cabeza R, Nyberg L (2000) Imaging cognition II: An empirical review of 275 PET and fMRI studies. J Cogn Neurosci 12: 1-47.

17. Kawashima R, Satoh K, Itoh H, Ono S, Furumoto S, et al. (1996) Functional anatomy of GO/NO-GO discrimination and response selection-a PET study in man. Brain Res 728: 79-89.

18. Garavan H, Ross TJ, Kaufman J, Stein EA (2003) A midline dissociation between error-processing and response-conflict monitoring. Neuroimage 20: 1132-1139.

19. Konishi S, Nakajima I, Kikyo H, Kameyama M, Miyashita Y (1999) Common inhibitory mechanism in human inferior prefrontal cortex revealed by event-related functional MRI. Brain 122: 981-991.

20. Tamm L, Menon V, Reiss AL (2002) Maturation of brain function associated with response inhibition. J Am Acad Child Adolesc Psychiatry 41: 1231-1238.

21. Terasawa K, Tabuchi H, Yanagisawa H, Yanagisawa A, Shinohara K, et al. (2014) Comparative survey of go/no-go results to identify the inhibitory control ability change of Japanese children. Biopsychosoc Med 8: 14.

22. Terasawa K, Misaki S, Murata Y, Watanabe T (2014) Relevance between Alzheimer's disease patients and normal subjects using go/no-go tasks and Alzheimer assessment scores. Journal of Child and Adolescent Behavior 2: 162.

23. Nakade K, Abe K, Fujiwara T, Terasawa K (2009) The influence of two different health education program on GO/NO-GO tasks, physical fitness tests and blood tests. Japan Society of Physical Anthropology 14: 143-150.

24. Murata Y, Nemoto K, Kobayashi I, Miyata Y (2015) Effect of a two-year health program on brain function, physical fitness and blood chemistry: Community Med \& Health Educ 5: 349. 